\title{
Modeling Brain Development Using Human Cells for the Study and Treatment of Zika Virus Infections
}

\author{
Kimberly M. Christian ${ }^{1,2}$
}

Published online: 3 November 2016

(C) Springer International Publishing AG 2016

\begin{abstract}
Purpose of Review Cellular reprogramming now permits the generation of a renewable source of disease-relevant human cells for modeling biological systems and for high-throughput drug screening. Advances in modeling human brain development to study Zika infections and its association with microcephaly are discussed in this commentary.

Recent Findings Human-induced pluripotent stem cells can be differentiated to cell types similar to those in the developing brain using both $2 \mathrm{D}$ and $3 \mathrm{D}$ cell culture systems. Zika virus targets proliferating neural progenitor cells in these systems and the resultant cell death provides evidence that Zika virus infections are causal for microcephaly. Drug repurposing screens using human cells vulnerable to infection has led to the identification of antiviral and neuroprotective therapeutic candidates. Summary Together with in vivo animal models, new stem cell-based technologies to model features of the developing and mature nervous system provide a platform for accelerated drug development to combat infectious diseases with neurological consequences.
\end{abstract}

Keywords Zika virus $\cdot$ Organoids $\cdot$ iPSCs $\cdot$ Disease modeling $\cdot$ Brain development

Kimberly M. Christian

kchris12@jhmi.edu

1 Institute for Cell Engineering, Johns Hopkins University School of Medicine, Baltimore, MD 21205, USA

2 Department of Neurology, Johns Hopkins University School of Medicine, Baltimore, MD 21205, USA
The recent outbreak of Zika virus (ZIKV) infections in dozens of countries throughout the Western Hemisphere has become a significant public health concern and was deemed a global emergency by the World Health Organization in February of 2016 [1]. Although ZIKV had been identified decades earlier, it was the striking coincidence of a rising number of microcephaly cases reported in regions with active transmission that triggered investigations of its effects on the developing nervous system. Within a few months, researchers from around the world mobilized to develop novel cellular and animal models to understand whether and how ZIKV infections in pregnant women may impact developing fetuses. What we have learned about ZIKV through this process is alarming in terms of its direct effects on human neural progenitors and its causal role in microcephaly. But how we reached these conclusions is encouraging in that it confirms we are at a transitional moment in biomedical research when we can exploit new technologies for rapid neurological disease modeling.

Neurodevelopmental disorders are among the most devastating and intractable of human diseases. Our understanding of the mechanisms underlying genetic or environmentally induced dysregulation of neural development has relied largely on indirect measures that must be interpreted with caution. Longitudinal studies of human brain development in patients and "at risk" populations are limited by the low resolution of non-invasive monitoring of brain structure and function. Postmortem examination of neural tissue cannot easily distinguish between causal pathology and its consequences. Animal models do offer distinct advantages in permitting the study of an intact nervous system, but are subject to a different set of constraints imposed by the lack of anatomical and genomic homology with humans. And, until recently, we had very little access to the most relevant human cell types to perform systematic investigations under controlled conditions in culture. 
The Nobel Prize-winning discovery that differentiated adult cells could be reprogrammed into stem cells has eliminated this last obstacle, with profound implications for diagnosing, treating, and preventing many disorders [2]. Cellular reprogramming allows for the derivation of induced pluripotent stem cell (iPSC) lines from any donor, which can then be banked and used as a renewable resource to generate any somatic cell type, including neurons and other cells of the central nervous system (CNS). For the first time, we can now produce an inexhaustible supply of human cells that share critical properties with the formative populations of the developing human brain, allowing for mechanistic investigations of disease and injuries affecting the nervous system.

Establishing a causal link between ZIKV and microcephaly, a disorder in which infants are born with small head sizes and stunted brain growth, required a better understanding of the cellular targets of ZIKV and the potential for vertical transmission from a pregnant woman to the fetus. Clinicians and researchers first detected the presence of ZIKV in fetal brains obtained from terminated pregnancies, demonstrating that the fetus was vulnerable to ZIKV [3, 4]. To determine whether ZIKV could directly infect the predominant cell type in the developing fetal brain, researchers turned to human neural progenitors differentiated from iPSCs. Strikingly, ZIKV preferentially targeted and induced cell death in these human neural proliferating cells, the population responsible for generating the cortical neurons that appeared to be the most affected in cases of microcephaly [5]. This result was cited in the report from scientists at the Centers for Disease Control and Prevention concluding that ZIKV was causal for microcephaly [6]. Although the demonstration that ZIKV could infect live human neural progenitors was critical, the fact that investigators can now probe the molecular and cellular machinery that mediate the toxic effect of ZIKV is even more important. It is precisely this ability to run repeated experiments, with appropriate controls, in disease-relevant cell types, that illustrates the power of iPSCs as a new tool for biological discovery and accelerated drug development.

The first reports of ZIKV infection of human neural progenitors came from studies using traditional 2-dimensional (2D) monolayer cell cultures. This standard approach offers several advantages such as the ability to generate highly pure populations of a specific cell type, well-defined stages of proliferation and differentiation synchronized across the population, and a fairly short developmental period until the cells reach maturity, as defined by functional properties and morphological features. Depending on the scientific question and the process being modeled, a potential disadvantage of the $2 \mathrm{D}$ system is its inability to capture essential aspects of organ development, namely the self-organization of multiple cell types at different developmental stages to produce highly structured 3D tissues.
In 2013, it was reported that culturing iPSCs in spinning bioreactors, under a pan-neural differentiation protocol that relied on intrinsic signaling cues, led to the formation of heterogeneous 3D cellular aggregates, or cerebral organoids, which expressed features of various brain regions [7]. Several groups recently used variations of this protocol to model ZIKV infections of brain development using cerebral organoids and obtained largely consistent results showing attenuated growth, increased cell death, and overall smaller organoids that were exposed to the virus [8-11]. Cerebral organoids allow for the identification of phenotypes potentially relevant to ZIKV that would not be apparent in 2D monolayer cultures, including dysregulated cell migration, cortical layer formation, and progenitor zone organization and function.

Ultimately, the goal of these ZIKV studies is to identify therapeutic targets to block or minimize the effects of ZIKV infections. Although ZIKV is also associated with cases of GuillainBarre syndrome in adults, the cerebral organoid system is ideally suited for modeling early brain development and microcephaly. Genome-wide expression analyses have been used to map the stages of organoid development to human gestational timepoints based on published transcriptome datasets, revealing analogous region-specific transcriptome dynamics up to the second trimester of pregnancy. A current technical challenge in the field is how to support maturation and growth of larger organoids to model later stages of brain development. After 80-100 days in culture, there is a physical limitation in the perfusion of growth factors and oxygen in the media to the center of the organoid, inducing cell death among the innermost layers and progenitor zones. Although current methodology permits the much-needed investigation of the earliest stages of brain development, prolonging culture conditions would allow for the study of chronic exposure and long-term effects of acute exposure of ZIKV on functional neural development.

Another challenge that is currently being addressed is the scalability of the organoid model to enable high-throughput screening that would facilitate rapid drug identification. Targeted mechanistic investigations of ZIKV based on observed phenotypes can lead to the development of hypothesis-driven rational drug development. A complementary approach is to perform unbiased screens of large compound libraries, with the robust phenotypes as a readout of drug efficacy. Using this latter strategy in a drug repurposing screen, both antiviral and neuroprotective compounds were identified that ameliorated viral production and death of human neural progenitors [12]. For urgent global health crises, the combination of drug screens using relevant human cell types and existing FDA-approved or clinical candidate drugs could dramatically decrease the time it takes to get a novel therapeutic to infected patients or populations most at risk. In addition to identifying a clear cellular phenotype for screening, reproducible and homogenous cell cultures are needed for quantification. To increase the throughput for organoid 
generation, a miniaturized bioreactor has been developed that allows for testing multiple conditions at a low cost. Optimized differentiation protocols will also be necessary to generate organoids with the salient properties of the system to be modeled, such as the presence of a human-specific outer subventricular zone that contributes to cortical development [10].

Organoids are highly relevant for understanding the acute effects of viral infections or other insults in early brain development. But an equally important question is how transient exposure to ZIKV in utero may affect the development and function of neural systems over the lifetime. Even in the absence of microcephaly or overt changes in global brain structure, there may be effects on the production or distribution of specific cell populations that alter brain circuitry, leading to cognitive deficits with varying degrees of severity. It is much too early to appreciate the long-term consequences of early ZIKV infections in affected human populations, but several groups have already reported viable animal models of ZIKV infection that will be essential to this research [11, 13-15]. It is clear that we need in vivo models to observe how ZIKV-induced perturbations of embryonic neurogenesis can influence the development of an intact nervous system and, ultimately, cognitive function and behavior.

Human iPSC-based research with 2D and 3D cell culture models cannot replace rodent or non-human primate models for discovery or evaluation of drug efficacy. But the promise and hope is that these human in vitro systems will expedite drug development and the identification of novel therapeutics through an approach that provides complementary advantages including the following: (1) higher fidelity models of human biological systems, (2) high-throughput assays using disease-relevant cell populations, (3) a rapidly deployable preclinical platform as an additional validation step before moving to clinical trials, and (4) donor-specific iPSC lines for diagnostics and to identify mechanisms underlying differences in susceptibility across the population.

Acknowledgments K.M.C. was partially supported by NIH grants NS097206 and MH106434.

\section{Compliance with Ethical Standards}

Conflict of Interest Dr. Kimberly Christian declares she has no conflict of interest.

Human and Animal Rights Informed Consent This article does not contain any studies with human or animal subjects performed by any of the authors.

\section{References}

1. Heymann DL, Hodgson A, Sall AA, Freedman DO, Staples JE, Althabe F, et al. Zika virus and microcephaly: why is this situation a PHEIC? Lancet. 2016;387(10020):719-21. doi:10.1016/S01406736(16)00320-2.

2. Takahashi K, Tanabe K, Ohnuki M, Narita M, Ichisaka T, Tomoda $\mathrm{K}$, et al. Induction of pluripotent stem cells from adult human fibroblasts by defined factors. Cell. 2007;131(5):861-72. doi:10.1016/j.cell.2007.11.019.

3. Driggers RW, Ho CY, Korhonen EM, Kuivanen S, Jaaskelainen AJ, Smura T, et al. Zika virus infection with prolonged maternal viremia and fetal brain abnormalities. N Engl J Med. 2016;374(22):214251. doi:10.1056/NEJMoa1601824.

4. Mlakar J, Korva M, Tul N, Popovic M, Poljsak-Prijatelj M, Mraz J, et al. Zika virus associated with microcephaly. N Engl J Med. 2016;374(10):951-8. doi:10.1056/NEJMoa1600651.

5. Tang H, Hammack C, Ogden SC, Wen Z, Qian X, Li Y, et al. Zika virus infects human cortical neural progenitors and attenuates their growth. Cell Stem Cell. 2016;18(5):587-90. doi:10.1016/j. stem.2016.02.016.

6. Rasmussen SA, Jamieson DJ, Honein MA, Petersen LR. Zika virus and birth defects - reviewing the evidence for causality. N Engl J Med. 2016;374(20):1981-7. doi:10.1056/NEJMsr1604338.

7. Lancaster MA, Renner M, Martin CA, Wenzel D, Bicknell LS, Hurles ME, et al. Cerebral organoids model human brain development and microcephaly. Nature. 2013;501(7467):373-9. doi:10.1038/nature12517.

8. Dang J, Tiwari SK, Lichinchi G, Qin Y, Patil VS, Eroshkin AM, et al. Zika virus depletes neural progenitors in human cerebral organoids through activation of the innate immune receptor TLR3. Cell Stem Cell. 2016;19(2):258-65. doi:10.1016/j. stem.2016.04.014.

9. Garcez PP, Loiola EC. Madeiro da Costa R, Higa LM, Trindade P, Delvecchio $\mathrm{R}$ et al. Zika virus impairs growth in human neurospheres and brain organoids. Science. 2016;352(6287):8168. doi:10.1126/science.aaf6116.

10. Qian X, Nguyen HN, Song MM, Hadiono C, Ogden SC, Hammack $\mathrm{C}$, et al. Brain-region-specific organoids using mini-bioreactors for modeling ZIKV exposure. Cell. 2016;165(5):1238-54. doi:10.1016 /j.cell.2016.04.032.

11. Cugola FR, Fernandes IR, Russo FB, Freitas BC, Dias JL, Guimaraes KP, et al. The Brazilian Zika virus strain causes birth defects in experimental models. Nature. 2016;534(7606):267-71. doi:10.1038/nature18296.

12. $\mathrm{Xu} \mathrm{M}$, Lee EM, Wen Z, Cheng Y, Huang WK, Qian X, et al. Identification of small-molecule inhibitors of Zika virus infection and induced neural cell death via a drug repurposing screen. Nat Med. 2016; doi:10.1038/nm.4184.

13. Dowall SD, Graham VA, Rayner E, Atkinson B, Hall G, Watson RJ, et al. A susceptible mouse model for Zika virus infection. PLoS Negl Trop Dis. 2016;10(5):e0004658. doi:10.1371/journal. pntd.0004658.

14. Li C, Xu D, Ye Q, Hong S, Jiang Y, Liu X, et al. Zika virus disrupts neural progenitor development and leads to microcephaly in mice. Cell Stem Cell. 2016;19(1):120-6. doi:10.1016/j.stem.2016.04.017.

15. Miner JJ, Cao B, Govero J, Smith AM, Fernandez E, Cabrera OH, et al. Zika virus infection during pregnancy in mice causes placental damage and fetal demise. Cell. 2016;165(5):1081-91. doi:10.1016 /j.cell.2016.05.008. 